\title{
Periodic Lyapunov functions for periodic TS systems
}

\author{
Zs. Lendek ${ }^{\mathrm{b}}$, J. Lauber ${ }^{\mathrm{a}}$, T. M. Guerra ${ }^{\mathrm{a}}$ \\ ${ }^{a}$ University of Valenciennes and Hainaut-Cambresis, LAMIH, \\ Le Mont Houy, 59313 Valenciennes Cedex 9, France. \\ ${ }^{b}$ Department of Automation, Technical University of Cluj-Napoca, \\ Memorandumului 28, 400114 Cluj-Napoca, Romania
}

\begin{abstract}
In this paper we consider stability analysis and controller design for periodic Takagi-Sugeno fuzzy models. To develop the conditions, we use a switching nonquadratic Lyapunov function defined at the time instants when the subsystems switch. Using the proposed conditions we are able to handle periodic Takagi-Sugeno systems where the local models or even the subsystems are unstable or cannot be stabilized. The application of the conditions is illustrated on numerical examples.
\end{abstract}

Keywords: Stability analysis, controller design, TS systems, periodic systems, non-quadratic Lyapunov function.

\section{Introduction}

Takagi-Sugeno (TS) fuzzy systems [1] are able to exactly represent a large class of nonlinear systems [2] as convex combinations of local linear models. They belong to the quasi- linear parameter varying (quasi-LPV) family.

Stability, controller, and observer design conditions for TS systems have been developed using the direct Lyapunov approach. Traditionally, conditions have been derived using quadratic Lyapunov functions [3] and piecewise quadratic Lyapunov functions [4]. More recently, to reduce the conservativeness of the conditions, nonquadratic Lyapunov functions [5] have been used. The stability or design conditions are usually formulated as linear matrix inequalities (LMIs).

In the discrete-time case, non-quadratic Lyapunov functions have shown a real improvement of the design conditions [6,7]. It has been proven that the solutions obtained by non-quadratic Lyapunov functions include and extend the set of solutions obtained using the quadratic framework.

To further improve the results, non-quadratic Lyapunov functions have been extended to double-sum Lyapunov functions [8] and later on to polynomial Lyapunov functions [9]. A different type of improvement in the discrete case is the $\alpha$-sample variation [10], using which the conditions are obtained by replacing the classical one sample variation of the Lyapunov function by its variation over several samples. 
This paper deals with a particular class of nonlinear models with periodic parameters which can be represented by periodic TS models. This kind of models can be found in numerous domains such as automotive, aeronautic, and aerospace or also computer control of industrial process. For example in [11], a periodic dynamic model is used to estimate the air/fuel ratio in each cylinder on an internal combustion engine, [12] proposes a periodic model for the rotor blades of helicopter, [13] deals with the problem of on-board automatic station keeping of a small spacecraft on a specific orbit of reference and proposes a periodic state feedback control law. Other examples are provided in [14] related to computer control and communication systems.

The stability of linear periodic systems is characterized by the monodromy transition matrix and by its eigenvalues, called the characteristic multipliers (often referred to the poles of the system). If all of the characteristic multipliers are in the open unit disc of the complex plane then the system is asymptotically stable [15]. Concerning the stabilization problem of those models, results are available in [16]. For models including time varying delays, [17] proposed methods based on Floquet's transformation, which is only applied to autonomous systems, and led to conditions for exponential stability.

Some extensions exist to polytopic LPV periodic models, where the stability analysis is based on the use of quadratic [16, 18] or non-quadratic [19] Lyapunov functions. In the nonlinear TS context, as far as we know, [20,21] are the only articles dealing with stabilization of discrete TS models with periodic parameters.

In this paper, we consider stability analysis and controller design for periodic, discrete-time TS models. To derive the conditions, we propose a periodic non-quadratic Lyapunov function. Although in general, stability analysis of TS models relies on the stability of each local model, and controller design for TS models requires the controllability of each local model, using this Lyapunov function we are able to prove the stability of a periodic TS system having non-stable local models and even unstable subsystems. Moreover, with the conditions derived using this Lyapunov function we are able to design stabilizing control laws for switching systems in the case when the local models of the subsystems are not stable and not stabilizable.

The structure of the paper is as follows. Section 2 presents a motivating example, the notations, and the existing results we make use of in this paper. The stability analysis of periodic TS systems, together with a discussion and an example is presented in Section 3. Section 4 presents and discusses the conditions for controller design and illustrates them on numerical examples. Section 5 concludes the paper.

\section{Preliminaries}

\subsection{A motivating example}

In the literature, one of the main assumptions on switching systems is that the switching can occur at any time, between any two subsystems. However, for periodic systems, the extra knowledge of when and between which subsystems the switching will occur can lead to more relaxed conditions. Consider for instance, the switching system composed of two linear 
subsystems, with state matrices

$$
A_{1}=\left(\begin{array}{cc}
0.7 & 0.8 \\
0.2 & 0.3
\end{array}\right) \quad A_{2}=\left(\begin{array}{cc}
0.5 & 0.8 \\
0.2 & a
\end{array}\right)
$$

where $a$ is a real-valued parameter. Using e.g., the results in [19], we are able to prove stability of the switching system for $a \in[-0.89,0.67]$. However, if we know that the system switches from one subsystem to the other at every time instant, the stability of the switching system can be proven for $a \in[-5.1,1.1]$.

Consequently, by using the knowledge of when and how a periodic system switches, can significantly relax the stability conditions. In what follows, we investigate this possibility for TS systems. Moreover, we extend the conditions to controller design, leading to considerable improvements of current design conditions.

\subsection{Background}

In this paper we consider stability analysis and controller design of discrete-time periodic TS systems. For stability analysis, we consider subsystems of the form

$$
\begin{aligned}
\boldsymbol{x}(k+1) & =\sum_{i=1}^{r_{j}} h_{j i}\left(\boldsymbol{z}_{j}(k)\right) A_{j, i} \boldsymbol{x}(k) \\
& =A_{j, z} \boldsymbol{x}(k)
\end{aligned}
$$

and for controller design

$$
\begin{aligned}
\boldsymbol{x}(k+1) & =\sum_{i=1}^{r_{j}} h_{j i}\left(\boldsymbol{z}_{j}(k)\right)\left(A_{j, i} \boldsymbol{x}(k)+B_{j, i} \boldsymbol{u}(k)\right) \\
& =A_{j, z} \boldsymbol{x}(k)+B_{j, z} \boldsymbol{u}(k)
\end{aligned}
$$

where $j$ is the number of the current subsystem, $j=1,2, \ldots, n_{\mathrm{s}}, n_{\mathrm{s}}$ being the number of the subsystems, $\boldsymbol{x}$ denotes the state vector, $r_{j}$ is the number of local models in the $j$ th subsystem, $\boldsymbol{z}_{j}$ is the scheduling vector, $h_{j i}, i=1,2, \ldots, r_{j}$ are normalized membership functions, and $A_{j, i}$ and $B_{j, i}, i=1,2, \ldots, r_{j}, j=1,2, \ldots, n_{\mathrm{s}}$, are the local models. We assume that the TS models do not have a finite escape time.

We consider periodic systems, i.e., the subsystems defined above are activated in a sequence $\underbrace{1,1, \ldots, 1}_{p_{1}}, \underbrace{2,2, \ldots, 2}_{p_{2}}, \ldots, \underbrace{n_{\mathrm{s}}, n_{\mathrm{s}}, \ldots, n_{\mathrm{s}}}_{p_{n_{\mathrm{s}}}}, \underbrace{1,1, \ldots, 1}_{p_{1}}$, etc., where $p_{i}$ denotes the number of samples for which the $i$ th subsystem is active. In what follows, we will refer to $p_{i}$ as the period of the $i$ th subsystem.

0 and $I$ denote the zero and identity matrices of appropriate dimensions, and a $(*)$ denotes the term induced by symmetry. The subscript $z+m$ (as in $A_{1, z+m}$ ) stands for the scheduling vector being evaluated at the current sample plus $m$ th instant, i.e., $\boldsymbol{z}_{1}(k+m)$. An underlined variable $\underline{j}$ denotes the modulo of the variable, i.e., $\underline{j}=\left(j \bmod n_{\mathrm{s}}\right)+1$. 
We use a Lyapunov function defined only in the switching instants. This also means that the $\alpha$-difference in the Lyapunov function corresponds to $\alpha$ consecutive switches in the system. To illustrate this, consider consider a switching TS model consisting of two subsystems, each with period 2, i.e., we have:

$$
\boldsymbol{x}(k+1)= \begin{cases}\sum_{i=1}^{r_{1}} h_{1 i}\left(\boldsymbol{z}_{1}(k)\right) A_{1 i} \boldsymbol{x}(k) & \text { if } k=4 m, 4 m+1 \\ \sum_{i=1}^{r_{2}} h_{2 i}\left(\boldsymbol{z}_{2}(k)\right) A_{2 i} \boldsymbol{x}(k) & \text { if } k=4 m+2,4 m+3\end{cases}
$$

The switching in the system and in the Lyapunov function are depicted in Figure 1. As can be seen, the Lyapunov function (with matrices $P_{1}$ and $P_{2}$ ) is defined only in the moments when there is a switching in the system: from $A_{1, z}$ to $A_{2, z}$ or from $A_{2, z}$ to $A_{1, z}$, respectively. A 1-sample variation of the Lyapunov function corresponds to the difference between two consecutive values of the Lyapunov function. A 2-sample variation corresponds to the difference after 2 samples of the Lyapunov function, etc.

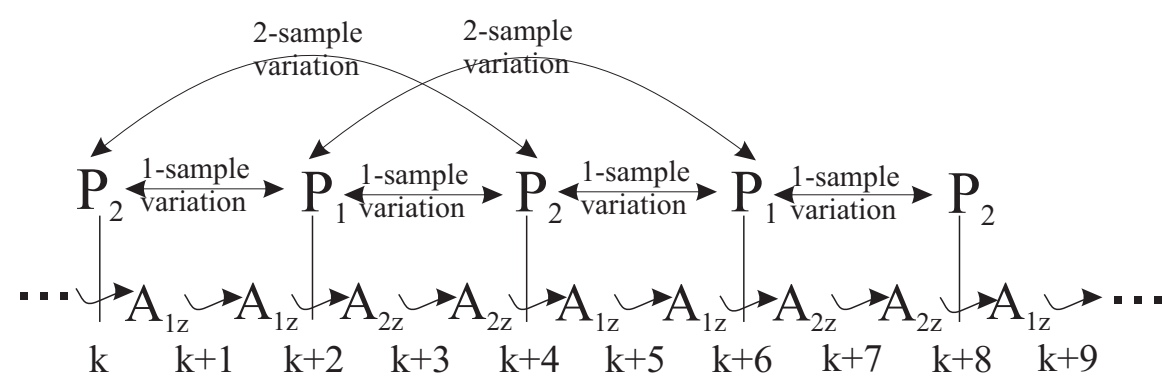

Figure 1: Switches in the system and in the Lyapunov function.

In what follows, we will make use of the following results:

Lemma 1. [22] Consider a vector $\boldsymbol{x} \in \mathbb{R}^{n_{x}}$ and two matrices $Q=Q^{T} \in \mathbb{R}^{n_{x} \times n_{x}}$ and $R \in$ $\mathbb{R}^{m \times n_{x}}$ such that $\operatorname{rank}(R)<n_{x}$. The two following expressions are equivalent:

1. $\boldsymbol{x}^{T} Q \boldsymbol{x}<0, \boldsymbol{x} \in\left\{\boldsymbol{x} \in \mathbb{R}^{n_{x}}, \boldsymbol{x} \neq 0, R \boldsymbol{x}=0\right\}$

2. $\exists M \in \mathbb{R}^{m \times n_{x}}$ such that $Q+M R+R^{T} M^{T}<0$

Controller design for TS models often leads to double-sum negativity problems of the form

$$
\boldsymbol{x}^{T} \sum_{i=1}^{r} \sum_{j=1}^{r} h_{i}(\boldsymbol{z}(k)) h_{j}(\boldsymbol{z}(k)) \Gamma_{i j} \boldsymbol{x}<0
$$

where $\Gamma_{i j}, i, j=1,2, \ldots, r$ are matrices of appropriate dimensions.

Lemma 2. [23] The double-sum (4) is negative, if

$$
\begin{aligned}
& \Gamma_{i i}<0 \\
& \frac{2}{r-1} \Gamma_{i i}+\Gamma_{i j}+\Gamma_{j i}<0, \quad i, j=1,2, \ldots, r, i \neq j
\end{aligned}
$$


Property 1. Let $A$ and $B$ be matrices of appropriate dimensions and ranks, with $B=B^{T}>$ 0. Then

$$
(A-B)^{T} B^{-1}(A-B) \geq 0 \Longleftrightarrow A^{T} B^{-1} A \geq A+A^{T}-B
$$

\section{Stability analysis of periodic TS systems}

\subsection{Stability conditions}

In this section, we consider the stability analysis of switching TS systems of the form (1). The results are also extended for $\alpha$-sample variation, similar to [10].

Consider the periodic TS system (1), composed of $n_{\mathrm{s}}$ subsystems, with each subsystem $j$ being active for $p_{j}$ samples, $j=1,2, \ldots, n_{\mathrm{s}}$. Then, the following results can be stated.

Theorem 1. The periodic TS system (1) with periods $p_{1}, p_{2}, \ldots, p_{n_{\mathrm{s}}}$ is asymptotically stable, if there exist $P_{j, i}=P_{j, i}^{T}>0, M_{j, i}, j=1,2, \ldots, n_{\mathrm{s}}, i=1,2, \ldots, r_{j}$, such that the following condition is satisfied:

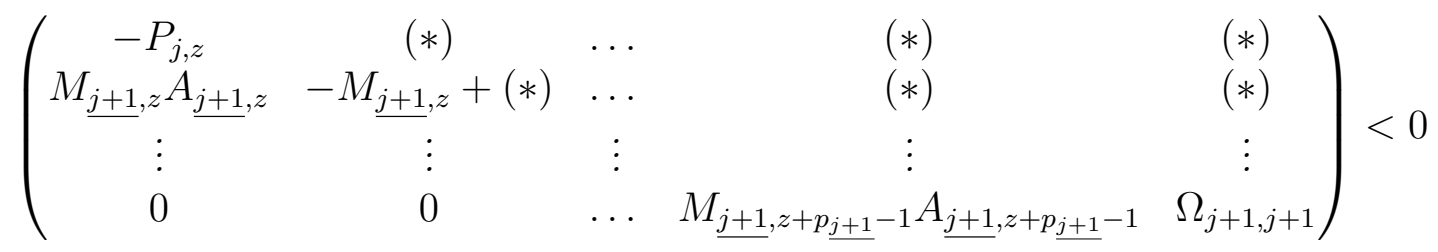

for $j=1,2, \ldots, n_{\mathrm{s}}$, where $\Omega_{j+1, j+1}=-M_{\underline{j+1, z+p_{j+1}-1}}+(*)+P_{\underline{j+1}, z+p_{j+1}}$.

Remark: Note that $j+1$ is used because due to the periodicity the $n_{\mathrm{s}}+i$ th subsystem is in fact the $i$ th one.

Proof: By considering a periodic Lyapunov function defined only in the instants when a switching takes place in the system:

$$
V(\boldsymbol{x}(k))=\boldsymbol{x}(k)^{T} P_{j, z} \boldsymbol{x}(k)
$$

the proof follows the same line of reasoning as the one of Theorem 3, Section 4.1.

Remark: Different from the control problem, LMI conditions may be obtained with virtually any multiplier matrix $M$, as long as the Lyapunov function uses $P_{j, z}$ instead of $P_{j, z}^{-1}$ as it is used in the control problem. However, to keep the notation simple, we only present the result for a specific case.

The result above can easily be extended using $\alpha$-sample variation of the Lyapunov function defined in the switching instant. Note that this means that the Lyapunov function does not have to decrease in every instant where it is defined, but it should decrease every $\alpha$ instants. Recall that the Lypunov function is only defined in the switching instants, and the $\alpha$-difference in the Lyapunov function corresponds to $\alpha$ consecutive switches in the system. Then, the following result can be formulated: 
Theorem 2. The periodic TS system (1) with periods $p_{1}, p_{2}, \ldots, p_{n_{\mathrm{s}}}$ is asymptotically stable, if there exist $P_{j, i}=P_{j, i}^{T}>0, M_{j, l, i}, j=1,2, \ldots, n_{\mathrm{s}}, i=1,2, \ldots, r_{j}, l=1,2, \ldots, \alpha$, such that the following condition is satisfied:

$$
\left(\begin{array}{cccccccc}
-P_{j, z} & (*) & \ldots & (*) & (*) & \ldots & (*) & (*) \\
\Gamma_{1,0} & -M_{\underline{j+1}, 1, z}+(*) & \ldots & (*) & (*) & \ldots & (*) & (*) \\
\vdots & \vdots & \vdots & \vdots & \vdots & \vdots & \vdots & \vdots \\
0 & 0 & \ldots & \Gamma_{2, p_{\underline{j+1}}} & -M_{\underline{j+1,2, z+p_{\underline{j+1}}}}+(*) & \ldots & (*) & (*) \\
\vdots & \vdots & \vdots & \vdots & \vdots & \vdots & \vdots & \vdots \\
0 & 0 & \ldots & 0 & 0 & \ldots & M_{\underline{j+1, \alpha, z+t-1}} A_{\underline{j+\alpha}, z+t-1} & \Omega_{j+\alpha, j+\alpha}
\end{array}\right)<0
$$

for $j=1,2, \ldots, n_{\mathrm{s}}$, where $t=\sum_{i=1}^{\alpha} \underline{p_{j+i}}, \Omega_{j+\alpha, j+\alpha}=-M_{\underline{j+1, \alpha, z+t-1}}-M_{\underline{j+1, \alpha, z+t-1}}^{T}+P_{\underline{j+\alpha, z+t-1}}$ and $\Gamma_{k, l}=M_{\underline{j+1, k, z+l}} A_{\underline{j+k, z+l}}$.

Proof: The proof is similar to that of Theorem 4, Section 4.1.

Remark: Similarly to the 1-sample variation, more general conditions may be obtained by using a general matrix $M$ in Lemma 1 .

\subsection{Examples and discussion}

First, let us discuss how exactly the conditions derived in Section 3.1 are applied. For simplicity, consider the switching TS model (3), consisting of two subsystems, each with period 2.

The switching in the system and in the Lyapunov function have been depicted in Figure 1.

For this system the conditions of Theorem 1 correspond to there exist $P_{j, i}=P_{j, i}^{T}>0, M_{j, i}$, $j=1,2, i=1,2, \ldots, r_{j}$, so that the following conditions are satisfied:

$$
\begin{aligned}
& \left(\begin{array}{ccc}
-P_{1, z} & (*) & (*) \\
M_{2, z} A_{2, z} & -M_{2, z}+(*) & (*) \\
0 & M_{2, z+1} A_{2, z+1} & -M_{2, z+1}+(*)+P_{2, z+2}
\end{array}\right)<0 \\
& \left(\begin{array}{ccc}
-P_{2, z} & (*) & (*) \\
M_{1, z} A_{1, z} & -M_{1, z}+(*) & (*) \\
0 & M_{1, z+1} A_{1, z+1} & -M_{1, z+1}+(*)+P_{1, z+2}
\end{array}\right)<0
\end{aligned}
$$

Relaxed LMI conditions can be formulated using Lemma 2, e.g.,

Corollary 1. The system (3) is asymptotically stable if there exist $P_{j, i}=P_{j, i}^{T}>0, M_{j, i}$, $j=1,2, i=1,2, \ldots, r_{j}$, so that

$$
\frac{2}{r_{\underline{l+1}}-1} \Gamma_{\text {liimno }}+\Gamma_{l j i m n o}+\Gamma_{\text {lijmno }}<0
$$

$i, j, l, m, n, o=1,2$, where

$$
\Gamma_{\text {lijmno }}=\left(\begin{array}{ccc}
-P_{l, i} & (*) & (*) \\
M_{\underline{l+1}, i} A_{\underline{l+1}, j} & -M_{\underline{l+1}, i}+(*) & (*) \\
0 & M_{\underline{l+1}, m} A_{\underline{l+1, n}, n} & -M_{\underline{l+1}, m}+(*)+P_{\underline{l+1}, o}
\end{array}\right)
$$


Let us now consider a 2-sample variation of the Lyapunov function. The conditions of Theorem 2 become there exist $P_{j, i}=P_{j, i}^{T}>0, M_{j, l, i}, j, l=1,2, i=1,2, \ldots, r_{j}$, so that the following conditions are satisfied:

$$
\begin{gathered}
\left(\begin{array}{ccccc}
-P_{1, z} & (*) & (*) & (*) & (*) \\
M_{2,1, z} A_{2, z} & -M_{2,1, z}+(*) & (*) & (*) & (*) \\
0 & M_{2,1, z+1} A_{2, z+1} & -M_{2,1, z+1}+(*) & (*) & (*) \\
0 & 0 & M_{2,2, z+2} A_{1, z+2} & -M_{2,2, z+2}+(*) & (*) \\
0 & 0 & 0 & M_{2,2, z+3} A_{1, z+3} & -M_{2,2, z+3}+(*)+P_{1, z+4}
\end{array}\right)<0 \\
\left(\begin{array}{ccccl}
-P_{2, z} & (*) & (*) & (*) & (*) \\
M_{1,1, z} A_{1, z} & -M_{1,1, z}+(*) & (*) & (*) & (*) \\
0 & M_{1,1, z+1} A_{1, z+1} & -M_{1,1, z+1}+(*) & (*) & (*) \\
0 & 0 & M_{1,2, z+2} A_{2, z+2} & -M_{1,2, z+2}+(*) & 0 \\
0 & 0 & 0 & M_{1,2, z+3} A_{2, z+3} & -M_{1,2, z+3}+(*)+P_{2, z+4}
\end{array}\right)<0
\end{gathered}
$$

Similarly to the 1-sample variation, relaxed LMI conditions can be formulated.

Although the number of samples to be used in the variation of the Lyapunov function can be chosen as $n_{s}$, this is not necessary. On the other hand, by increasing $\alpha$, the number and the dimension of the LMIs to be solved increases.

In developing the conditions, in Finsler's lemma we used the same matrices in the multiplication of the same subsystem, i.e., we have $M_{1} A_{1}, M_{2} A_{2}$, etc., even for different time instants. Different matrices can be used at each time instant, or even a completely general multiplier can be used in Lemma 1, but with the cost of significantly increasing the number of decision variables. As it is, the number of decision variables is $\sum_{i=1}^{n_{\mathrm{s}}} r_{i} n_{x}^{2}+\sum_{i=1}^{n_{\mathrm{s}}} r_{i} n_{x}\left(n_{x}+1\right) / 2$, where $n_{x}$ denotes the dimension of the state. It should be noted that the number of decision variables depends only on the number of the subsystems, the number of rules in each subsystem, and the dimension of the state. The number and the dimension of the actual LMIs to be solved depends on the relaxation used.

Note that the conditions do not require that the local matrices of the TS systems or even the individual subsystems are stable. We illustrate this on the following example.

Example 1. Consider the switching fuzzy system with two subsystems as follows:

$$
\boldsymbol{x}(k+1)=\sum_{i=1}^{2} h_{1 i}\left(\boldsymbol{z}_{1}(k)\right) A_{1 i} \boldsymbol{x}(k)
$$

with

$$
A_{11}=\left(\begin{array}{cc}
-0.44 & -0.26 \\
-0.65 & 0.62
\end{array}\right) \quad A_{12}=\left(\begin{array}{cc}
1.1 & -0.2 \\
0.53 & -0.27
\end{array}\right)
$$

with $h_{11}=\exp \left(-x_{1}^{2}\right), h_{12}=1-h_{11}$ and

$$
\boldsymbol{x}(k+1)=\sum_{i=1}^{2} h_{2 i}\left(\boldsymbol{z}_{1}(k)\right) A_{2 i} \boldsymbol{x}(k)
$$




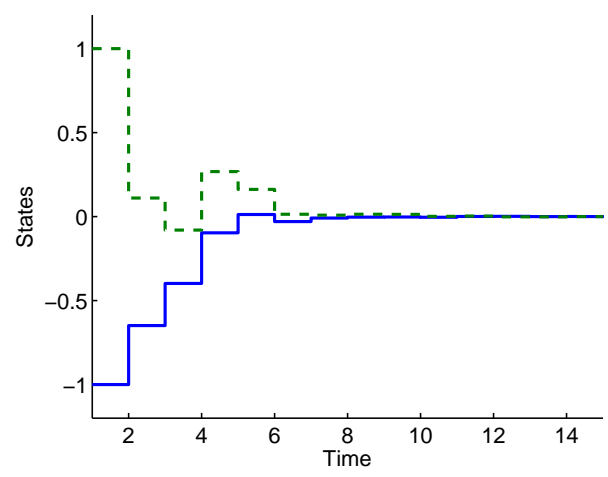

Figure 2: A trajectory of the states of the switching system.

with

$$
A_{21}=\left(\begin{array}{cc}
0.02 & 0.6 \\
-0.22 & -0.44
\end{array}\right) \quad A_{22}=\left(\begin{array}{cc}
0.32 & -0.15 \\
-1 & 0.8
\end{array}\right)
$$

with $h_{21}=\cos \left(x_{1}\right)^{2}, h_{22}=1-h_{21}$.

The local models $A_{12}$ and $A_{22}$ are unstable, their eigenvalues being $(1.0177-0.1877)$ and (0.1044 1.0156), respectively.

By switching between the two subsystems with a period $p_{1}=2$ for the first subsystem and $p_{2}=2$ for the second subsystem, the resulting switching system is asymptotically stable, as illustrated in Figure 2.

For this switching system it is not possible to find either a quadratic or a nonquadratic Lyapunov function, common for both subsystems, as the corresponding LMIs are unfeasible.

The conditions of Theorem 1 are those in (6). Solving them using the relaxation of [24], we obtain

$$
\begin{array}{rlr}
P_{11}=\left(\begin{array}{cc}
2.7195 & -0.6733 \\
-0.6733 & 3.7396
\end{array}\right) & P_{12}=\left(\begin{array}{cc}
3.9735 & -1.7562 \\
-1.7562 & 3.6588
\end{array}\right) \\
M_{11}=\left(\begin{array}{cc}
4.4862 & 0.0610 \\
-0.0699 & 3.3215
\end{array}\right) & M_{12}=\left(\begin{array}{cc}
4.2601 & 0.6030 \\
-0.3073 & 2.5510
\end{array}\right) \\
P_{21}=\left(\begin{array}{cc}
4.3030 & 0.0691 \\
0.0691 & 2.1985
\end{array}\right) & P_{22}=\left(\begin{array}{cc}
5.2906 & 0.0310 \\
0.0310 & 1.9130
\end{array}\right) \\
M_{21}=\left(\begin{array}{cc}
3.9873 & -0.6752 \\
-0.6623 & 3.5612
\end{array}\right) & M_{22}=\left(\begin{array}{cc}
3.5420 & -1.0998 \\
-0.7195 & 3.4535
\end{array}\right)
\end{array}
$$

\section{Controller design}

\subsection{Design conditions}

In the previous section, we have considered stability analysis of periodic systems. Now we extend the obtained results to controller design for periodic TS systems. 
Consider the switching TS model (2), consisting of $n_{\mathrm{s}}$ subsystems, each having the period (being active for) $p_{i}$ samples, $i=1,2 \ldots, n_{\mathrm{s}}$. For this system, we use the switching control input of the form

$$
\boldsymbol{u}(k)=-F_{i, z} H_{i, z}^{-1} \boldsymbol{x}(k)
$$

when the $i$ th subsystem is active, $i=1,2, \ldots, n_{\mathrm{s}}$. The switching closed-loop dynamics are

$$
\boldsymbol{x}(k+1)=\left(A_{i, z}-B_{i, z} F_{i, z} H_{i, z}^{-1}\right) \boldsymbol{x}(k)
$$

For (9) the following result can be stated:

Theorem 3. The periodic TS system (1) with periods $p_{1}, p_{2}, \ldots, p_{n_{\mathrm{s}}}$ is asymptotically stabilized by the control input (8), if there exist $P_{j, i}=P_{j, i}^{T}>0, F_{j, i}, H_{j, i} j=1,2, \ldots, n_{\mathrm{s}}$, $i=1,2, \ldots, r_{j}$, such that the following condition is satisfied:

$$
\left(\begin{array}{ccccc}
-H_{\underline{j+1, z}}-H_{\underline{j+1, z}}^{T}+P_{j, z} & (*) & \ldots & (*) & (*) \\
\Omega_{j+1,1} & -H_{\underline{j+1, z+1}}+(*) & \ldots & (*) & (*) \\
\vdots & \vdots & \vdots & \vdots & \vdots \\
0 & 0 & \ldots & \Omega_{j+1, p_{\underline{j+1}}} & -P_{\underline{j+1, z+p_{\underline{j+1}}}}
\end{array}\right)<0
$$

for $j=1,2, \ldots, n_{\mathrm{s}}$, where $\Omega_{j+1, l}=A_{\underline{j+1, z+l-1}} H_{\underline{j+1, z+l-1}}-B_{\underline{j+1, z+l-1}} F_{\underline{j+1}, z+l-1}$.

Proof: Consider the switching Lyapunov function, defined only in the instants when a switching takes place in the system:

$$
V(\boldsymbol{x}(k))=\boldsymbol{x}(k)^{T} P_{j, z}^{-1} \boldsymbol{x}(k)
$$

if the active subsystem was $j, j=1,2 \ldots, n_{\mathrm{s}}$.

The difference in the Lyapunov function is

$$
\begin{aligned}
& V\left(\boldsymbol{x}\left(k+p_{\underline{j+1}}\right)\right)-V(\boldsymbol{x}(k))= \\
& \left(\begin{array}{c}
\boldsymbol{x}(k) \\
\boldsymbol{x}\left(k+p_{\underline{j+1}}\right)
\end{array}\right)^{T}\left(\begin{array}{cc}
-P_{j, z}^{-1} & 0 \\
0 & P_{\underline{j+1}, z+p_{\underline{j+1}}}^{-1}
\end{array}\right)\left(\begin{array}{c}
\boldsymbol{x}(k) \\
\boldsymbol{x}\left(k+p_{\underline{j+1}}\right)
\end{array}\right)
\end{aligned}
$$

The closed-loop system dynamics during the $p_{\underline{j+1}}$ samples are

$$
\left(\begin{array}{ccccc}
\Upsilon_{j+1,1} & -I & \ldots & 0 & 0 \\
0 & \Upsilon_{j+1,2} & -I & \ldots & 0 \\
\vdots & \vdots & \vdots & \vdots & \vdots \\
0 & 0 & \ldots & \Upsilon_{j+1, p_{j+1}-1} & -I
\end{array}\right)\left(\begin{array}{c}
\boldsymbol{x}(k) \\
\boldsymbol{x}(k+1) \\
\vdots \\
\boldsymbol{x}\left(k+p_{\underline{j+1}}\right)
\end{array}\right)=0
$$

with

$$
\Upsilon_{j+1, i}=A_{\underline{j+1, z+i}}-B_{\underline{j+1, z+i}} F_{\underline{j+1}, z+i} H_{\underline{j+1}, z+i}^{-1}
$$


for $i=1,2 \ldots, p_{j+1}-1$.

$$
\text { Choosing } M=\left(\begin{array}{ccccc}
0 & 0 & \ldots & 0 & 0 \\
H_{\underline{j+1}, z+1}^{-T} & 0 & \ldots & 0 & 0 \\
0 & H_{\underline{j+1, z+2}}^{-T} & \ldots & 0 & 0 \\
\vdots & \vdots & \vdots & \vdots & \vdots \\
0 & 0 & \ldots & H_{\underline{j+1, z+p_{j+1}-1}}^{T} & 0 \\
0 & 0 & \ldots & 0 & P_{\underline{j+1, z+p_{j+1}}}^{-1}
\end{array}\right) \text { and applying Lemma } 1
$$

leads to

$$
\left(\begin{array}{ccccc}
-P_{j z}^{-1} & (*) & \ldots & (*) & (*) \\
\Omega_{j+1,1} & -H_{j+1, z+1}^{-1}+(*) & \ldots & (*) & (*) \\
\vdots & \vdots & \vdots & \vdots & \vdots \\
0 & 0 & \ldots & \Omega_{j+1, \underline{p_{j+1}}} & -P_{j+1, z+p_{\underline{j+1}}}^{-1}
\end{array}\right)<0
$$

with $\Omega_{j+1, i}=H_{\underline{j+1}, z+i}^{-T}\left(A_{\underline{j+1}, z+i-1}-B_{\underline{j+1}, z+i-1} F_{\underline{j+1, z+i-1}} H_{\underline{j+1}, z+i-1}^{-1}\right)$ for $i=1,2, \ldots, p_{j+1}-1$, $\left.\Omega_{j+1, t}=P_{j+1, z+t}^{-T} \overline{(A}_{\underline{j+1, z+t-1}}-B_{\underline{j+1, z+t-1}} F_{\underline{j+1, z+t-1}} H_{j+1, z+t-1}^{-1}\right)$.

Congruence with

$$
\left(\begin{array}{ccccc}
H_{\underline{j+1, z}}^{T} & 0 & \cdots & 0 & 0 \\
0 & H_{\underline{j+1, z+1}}^{T} & \cdots & 0 & 0 \\
\vdots & \vdots & \vdots & \vdots & \vdots \\
0 & 0 & \ldots & H_{\underline{j+1, z+p_{j+1}-1}}^{T} & 0 \\
0 & 0 & \cdots & 0 & P_{\underline{j+1, z+p_{\underline{j+1}}}}
\end{array}\right)
$$

and applying Property 1 leads directly to (10).

In what follows, we extend the above result to $\alpha$-sample variation [10]. When $\alpha$-sample variation of the Lyapunov function is considered, the following result can be stated.

Theorem 4. The periodic TS system (1) with periods $p_{1}, p_{2}, \ldots, p_{n_{\mathrm{s}}}$ is asymptotically stable, if there exist $P_{j, i}=P_{j, i}^{T}>0, F_{j, i}$, and $H_{j, i}, j=1,2, \ldots, n_{\mathrm{s}}, i=1,2, \ldots, r_{j}, l=1,2, \ldots, \alpha$, such that the following condition is satisfied:

$$
\left(\begin{array}{ccccc}
-H_{\underline{j+1, z}}-H_{\underline{j+1, z}}^{T}+P_{j, z} & (*) & \ldots & (*) & (*) \\
\Omega_{j+1,1} & -H_{\underline{j+1, z+1}}+(*) & \ldots & (*) & (*) \\
\vdots & \vdots & \vdots & \vdots & \vdots \\
0 & 0 & \ldots & \Omega_{j+\alpha, \underline{\underline{j+1}}} & -P_{\underline{j+\alpha, z+t}}
\end{array}\right)<0
$$

for $j=1,2, \ldots, n_{\mathrm{s}}$, where $\Omega_{j+i, l}=A_{\underline{j+i, z+l-1}} H_{\underline{j+i, z+l-1}}-B_{\underline{j+i, z+l-1}} F_{\underline{j+i, z+l-1}}, i=1,2, \ldots, \alpha$, $l=1,2, \ldots, t$, and $t=\sum_{i=1}^{\alpha} p_{\underline{j+i}}$.

Proof: The proof follows the same line as that of Theorem 3. 


\subsection{Examples and discussion}

Let us discuss first how exactly the controller design conditions derived in Section 4.1 are applied. Consider a switching TS model consisting of two subsystems, each with period 2, and each having two rules, i.e., we have:

$$
\boldsymbol{x}(k+1)= \begin{cases}\sum_{i=1}^{2} h_{1 i}\left(\boldsymbol{z}_{1}(k)\right)\left(A_{1 i} \boldsymbol{x}(k)+B_{1 i} \boldsymbol{u}(k)\right) & \text { if } k=4 m, 4 m+1 \\ \sum_{i=1}^{2} h_{2 i}\left(\boldsymbol{z}_{2}(k)\right)\left(A_{2 i} \boldsymbol{x}(k)+B_{2 i} \boldsymbol{u}(k)\right) & \text { if } k=4 m+2,4 m+3\end{cases}
$$

For the system (12) the conditions of Theorem 3 correspond to there exist $P_{j, i}=P_{j, i}^{T}>0$, $H_{j, i}$, and $F_{j, i}, j, i=1,2$, so that the following conditions are satisfied:

$$
\begin{gathered}
\left(\begin{array}{ccc}
-H_{2, z}+(*)+P_{1, z} & (*) & (*) \\
A_{2, z} H_{2, z}-B_{2, z} F_{2, z} & -H_{2, z+1}+(*) & (*) \\
0 & A_{2, z+1} H_{2, z+1}-B_{2, z+1} F_{2, z+1} & -P_{2, z+2}
\end{array}\right)<0 \\
\left(\begin{array}{ccc}
-H_{1, z}+(*)+P_{2, z} & (*) & (*) \\
A_{1, z} H_{1, z}-B_{1, z} F_{1, z} & -H_{1, z+1}+(*) & (*) \\
0 & A_{1, z+1} H_{1, z+1}-B_{1, z+1} F_{1, z+1} & -P_{1, z+2}
\end{array}\right)<0
\end{gathered}
$$

The conditions of Theorem 4, e.g., for a 2-sample variation become there exist $P_{j, i}=P_{j, i}^{T}>$ $0, H_{j, i}$, and $F_{j, i}, j, i=1,2$, so that the following conditions are satisfied:

$$
\begin{aligned}
& \left(\begin{array}{ccccc}
-H_{2, z}+(*)+P_{1, z} & (*) & (*) & (*) & (*) \\
\Omega_{21} & -H_{2, z+1}+(*) & (*) & (*) & (*) \\
0 & \Omega_{22} & -H_{1, z+2}+(*) & (*) & (*) \\
0 & 0 & \Omega_{13} & -H_{1, z+3}+(*) & (*) \\
0 & 0 & 0 & \Omega_{14} & -P_{1, z+4}
\end{array}\right)<0 \\
& \left(\begin{array}{ccccc}
-H_{1, z}+(*)+P_{2, z} & (*) & (*) & (*) & (*) \\
\Omega_{11} & -H_{1, z+1}+(*) & (*) & (*) & (*) \\
0 & \Omega_{12} & -H_{2, z+2}+(*) & (*) & (*) \\
0 & 0 & \Omega_{23} & -H_{2, z+3}+(*) & (*) \\
0 & 0 & 0 & \Omega_{24} & -P_{2, z+4}
\end{array}\right)<0
\end{aligned}
$$

As illustrated in the above conditions, for each subsystem we have a matrix inequality. In the $i$ th matrix inequality, the first line corresponds to $P_{i, z}$, the next $p_{i+1}$ lines correspond to the $i+1$ th subsystems, etc.

As can be expected based on the stability conditions, in order to the closed-loop switching system to be stable it is not necessary that each subsystem is stabilized. We illustrate this on the following example, adopted from [20]. 
Example 2. Consider the periodic fuzzy system, composed of two subsystems, with the local matrices:

$$
\begin{array}{ll}
A_{11}=\left(\begin{array}{cc}
1.5 & 10 \\
0 & 0.5
\end{array}\right) & A_{12}=\left(\begin{array}{cc}
0.5 & 10 \\
0 & 0
\end{array}\right) \\
A_{21}=\left(\begin{array}{cc}
1+a & 1 \\
0 & 0.5
\end{array}\right) & A_{22}=\left(\begin{array}{cc}
1 & 10 \\
0 & 0.5
\end{array}\right) \\
B_{11}=B_{12}=\left(\begin{array}{l}
0 \\
0
\end{array}\right) & B_{21}=B_{22}=\left(\begin{array}{l}
1 \\
0
\end{array}\right)
\end{array}
$$

where ' $a$ ' is a real-valued parameter.

The control design for this system cannot be performed using a Lyapunov function that is common for both subsystems, be that quadratic or nonquadratic. In the switching system above, the first subsystem is not stabilizable, as $B_{11}=B_{12}=0$, and the first local model $A_{11}$ is unstable.

With the approach of [20], the maximum interval for ' $a$ ' that can be obtained is [-250,250]. Using the conditions of Theorem 3, we obtain for $a=1500$

$$
\begin{aligned}
& H_{11}=\left(\begin{array}{cc}
240.8746 & -33.2523 \\
-34.5795 & 10.3966
\end{array}\right) \quad H_{12}=\left(\begin{array}{cc}
239.2061 & -37.6113 \\
-11.2734 & 7.4435
\end{array}\right) \\
& H_{21}=\left(\begin{array}{cc}
374.8946 & 8.7529 \\
0.0039 & 33.2571
\end{array}\right) \quad H_{22}=\left(\begin{array}{cc}
374.8945 & 8.7468 \\
-0.0039 & 33.2570
\end{array}\right) \\
& P_{11}=\left(\begin{array}{cc}
561.3074 & 8.7531 \\
8.7531 & 18.2803
\end{array}\right) \quad P_{12}=\left(\begin{array}{cc}
561.3065 & 8.7466 \\
8.7466 & 18.2802
\end{array}\right) \\
& P_{21}=\left(\begin{array}{cc}
295.0400 & -45.1654 \\
-45.1654 & 9.8090
\end{array}\right) \quad P_{22}=\left(\begin{array}{cc}
300.2630 & -38.8747 \\
-38.8747 & 7.7637
\end{array}\right) \\
& F_{21}=10^{5}\left(\begin{array}{ll}
5.6272 & 0.1325
\end{array}\right) \quad F_{22}=\left(\begin{array}{lll}
374.8512 & 417.8578
\end{array}\right)
\end{aligned}
$$

which stabilizes the system. Moreover, the value of ' $a$ ' can still be increased. Trajectories of the closed-loop system for $a=1500$ are shown in Figure 3. The membership functions used were $h_{11}=\exp \left(-x_{1}^{2}\right), h_{12}=1-h_{11}$ and $h_{21}=\cos \left(x_{1}\right)^{2}, h_{22}=1-h_{21}$.

To illustrate the efficiency of the proposed method, consider now a more complex example.

Example 3. Consider the periodic fuzzy system, composed of 5 subsystems, with the local 


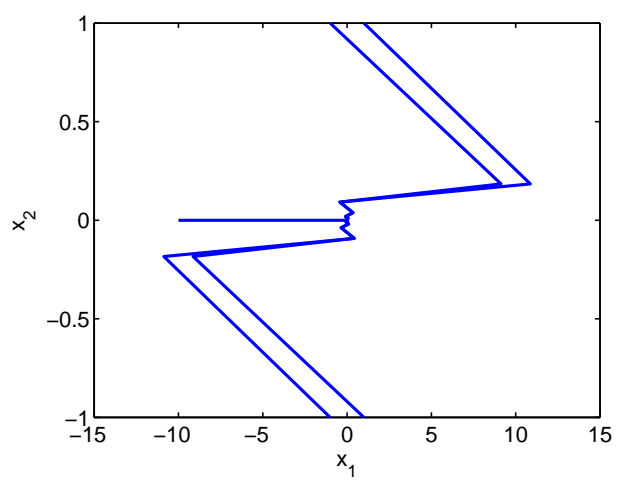

Figure 3: Trajectories of the closed-loop system - example 2.

matrices:
$A_{11}=\left(\begin{array}{ll}2 & 0 \\ 0 & 2\end{array}\right)$
$A_{12}=\left(\begin{array}{ll}2 & 0 \\ 0 & 2\end{array}\right)$
$B_{11}=\left(\begin{array}{l}0 \\ 0\end{array}\right)$
$B_{12}=\left(\begin{array}{l}1 \\ 0\end{array}\right)$
$A_{21}=\left(\begin{array}{cc}1.5 & 1 \\ 0 & 1.5\end{array}\right)$
$A_{22}=\left(\begin{array}{cc}1.5 & 1 \\ 0 & 1.5\end{array}\right)$
$B_{21}=\left(\begin{array}{l}0 \\ 1\end{array}\right)$
$B_{22}=\left(\begin{array}{l}0 \\ 0\end{array}\right)$
$A_{31}=\left(\begin{array}{ll}1 & 0 \\ 0 & 2\end{array}\right)$
$A_{32}=\left(\begin{array}{cc}1.5 & 0 \\ 0 & .5\end{array}\right)$
$B_{31}=\left(\begin{array}{l}1 \\ 2\end{array}\right)$
$B_{32}=\left(\begin{array}{l}1 \\ 2\end{array}\right)$
$A_{41}=\left(\begin{array}{cc}-0.44 & -0.26 \\ -0.65 & 0.62\end{array}\right)$
$A_{42}=\left(\begin{array}{cc}1.1 & -0.2 \\ 0.53 & -0.27\end{array}\right)$
$B_{41}=\left(\begin{array}{l}1 \\ 0\end{array}\right)$
$B_{42}=\left(\begin{array}{l}1 \\ 0\end{array}\right)$
$A_{51}=\left(\begin{array}{cc}1.32 & -0.15 \\ -1 & 0.8\end{array}\right)$
$A_{52}=\left(\begin{array}{cc}0.02 & 1.6 \\ -0.22 & -0.44\end{array}\right)$
$B_{51}=\left(\begin{array}{c}1 \\ -1\end{array}\right)$
$B_{52}=\left(\begin{array}{c}1 \\ -1\end{array}\right)$

and periodicity $p=[1,1,1,2,2]$.

Again, several local models are not controllable and not stable, and consequently the control design cannot be performed using a Lyapunov function that is common for both subsystems, be that quadratic or nonquadratic. However, by using a switching control law, with a 1-sample variation, the system is stabilized. A trajectory of the closed-loop system is shown in Figure 4. To design the controller, $184 \times 4$ and $366 \times 6$ LMIs have been solved, and 90 decision variables have been computed.

Similarly to the results in Section 3, relaxed LMI conditions can be obtained. The number of decision variables in the LMIs for the 1 -sample variation is $\sum_{i=1}^{n_{\mathrm{s}}} r_{i}\left(n_{x}^{2}+n_{x}\left(n_{x}+1\right) / 2+n_{x} n_{u}\right)$, where $n_{x}$ denotes the dimension of the state and $n_{u}$ the dimension of the input.

Compared to the possibility in case of stability conditions, the freedom in choosing the multiplier in Lemma 1 is much smaller. This is because the controller is fixed for each subsystem. Also in this case, the number of decision variables depends only on the number 


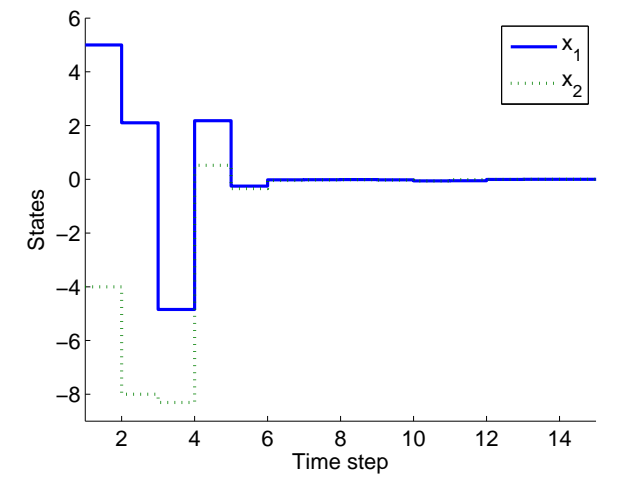

Figure 4: A trajectory of the closed-loop system - example 3.

of the subsystems, the number of rules in each subsystem, and the dimension of the state and input. The number and the dimension of the actual LMIs to be solved depends on the relaxation used and on the sample-variation used, as by increasing $\alpha$, the number and dimension of the LMIs to be solved increases.

Let us illustrate this on the following example:

Example 4. Consider a periodic fuzzy system consisting of 3 subsystems, each with 2 rules, 2 states, and 1 input. A comparison of the number and dimension of the LMIs to be solved for controller design for different periodicity of the subsystems and different sample variation, using the relaxation of [24] on all possible pairs is given in Table 1. The number of decision variables is $\sum_{i=1}^{n_{\mathrm{s}}} r_{i}\left(n_{x}^{2}+n_{x}\left(n_{x}+1\right) / 2+n_{x} n_{u}\right)=54$, in all the cases.

As can be seen, with a higher $\alpha$-sample variation used, the number of LMIs and their dimension quickly increases.

\section{Conclusions}

In this paper we have developed conditions for the stability analysis and stabilization of periodic TS systems. We considered a periodic controller and we used a periodic Lyapunov function, defined in the points where the subsystems switch. Using the developed conditions, one is able to design a controller for a system where the local models are not stable and not controllable. In our future work, we will reduce the conservatism of the developed conditions.

\section{Acknowledgment}

This work was supported by a grant of the Romanian National Authority for Scientific Research, CNCS - UEFISCDI, project number PN-II-RU-TE-2011-3-0043, contract number 74/05.10.2011, by International Campus on Safety and Intermodality in Transportation the European Community, 
Table 1: Comparison of number of LMIs

\begin{tabular}{lccc}
\hline Periods & Samples & Number & Dimension \\
\hline $1,1,1$ & 1 & 18 & $4 \times 4$ \\
$1,1,2$ & 1 & 12 & $4 \times 4$ \\
& & 18 & $6 \times 6$ \\
$1,2,2$ & 1 & 36 & $6 \times 6$ \\
$2,2,2$ & 1 & 54 & $4 \times 4$ \\
$2,2,3$ & 1 & 36 & $6 \times 6$ \\
& & 54 & $8 \times 8$ \\
$2,3,3$ & 1 & 18 & $6 \times 6$ \\
& & 108 & $8 \times 8$ \\
\hline $1,1,1$ & 2 & 54 & $6 \times 6$ \\
$1,1,2$ & 2 & 18 & $6 \times 6$ \\
& & 108 & $8 \times 8$ \\
$1,2,2$ & 2 & 162 & $10 \times 10$ \\
$2,2,2$ & 2 & 486 & $10 \times 10$ \\
\hline & & & \\
$1,1,1$ & 3 & 162 & $8 \times 8$ \\
$1,1,2$ & 3 & 486 & $10 \times 10$ \\
\hline
\end{tabular}

the Délegation Régionale à la Recherche et à la Technologie, the Ministére de L'Enseignement supérieur et de la Recherche the Region Nord Pas de Calais and the Centre Nationale de la Recherche Scientifique: the authors gratefully acknowledge the support of these institutions.

\section{References}

[1] T. Takagi, M. Sugeno, Fuzzy identification of systems and its applications to modeling and control, IEEE Transactions on Systems, Man, and Cybernetics 15 (1) (1985) 116-132.

[2] Zs. Lendek, T. M. Guerra, R. Babuška, B. De Schutter, Stability analysis and nonlinear observer design using Takagi-Sugeno fuzzy models, Vol. 262 of Studies in Fuzziness and Soft Computing, Springer Germany, 2010.

[3] K. Tanaka, H. O. Wang, Fuzzy Control System Design and Analysis: A Linear Matrix Inequality Approach, John Wiley \& Sons, New York, NY, USA, 2001.

[4] M. Johansson, A. Rantzer, K. Arzen, Piecewise quadratic stability of fuzzy systems, IEEE Transactions on Fuzzy Systems 7 (6) (1999) 713-722.

[5] T. M. Guerra, L. Vermeiren, LMI-based relaxed nonquadratic stabilization conditions for nonlinear systems in the Takagi-Sugeno's form, Automatica 40 (5) (2004) 823-829. 
[6] D. H. Lee, J. B. Park, Y. H. Joo, Approaches to extended non-quadratic stability and stabilization conditions for discrete-time Takagi-Sugeno fuzzy systems, Automatica 47 (3) (2011) $534-538$.

[7] T. M. Guerra, H. Kerkeni, J. Lauber, L. Vermeiren, An efficient Lyapunov function for discrete TS models: observer design, IEEE Transactions of Fuzzy Systems 20 (1) (2012) 187 - 192.

[8] B. Ding, H. Sun, P. Yang, Further studies on LMI-based relaxed stabilization conditions for nonlinear systems in Takagi-Sugeno's form, Automatica 42 (3) (2006) 503-508.

[9] A. Sala, C. Ariño, Asymptotically necessary and sufficient conditions for stability and performance in fuzzy control: Applications of Polya's theorem, Fuzzy Sets and Systems 158 (24) (2007) 2671-2686.

[10] A. Kruszewski, R. Wang, T. M. Guerra, Nonquadratic stabilization conditions for a class of uncertain nonlinear discrete time TS fuzzy models: A new approach, IEEE Transactions on Automatic Control 53 (2) (2008) 606-611.

[11] J. Chauvin, P. Moulin, G. Corde, N. Petit, P. Rouchon, Real-time nonlinear individual cylinder air-fuel ratio observer on a diesel engine test bench, in: Preprints of the IFAC World Congress, Prague, Czech Republic, 2005.

[12] G. Gaiani, M. Lovera, P. Colaneri, R. Celi., Discrete-time analysis of HHC schemes for helicopter vibration attenuation, in: Proceedings of the IFAC Workshop on Periodic Control Systems, Yokohama, Japan, 2004, pp. 69-74.

[13] A. Theron, C. Farges, D. Peaucelle, D. Arzelier, Periodic $H_{2}$ synthesis for spacecraft in elliptical orbits with atmospheric drag and perturbations, in: Proceedings of American Control Conference, New York, USA, 2007.

[14] S. Bittanti, P. Colaneri, Invariant representations of discrete-time periodic systems, Automatica 36 (2000) 1777-1793.

[15] M. Farkas, Periodic motions., New York: Springer-Verlag, 1994.

[16] C. Farges, D. Peaucelle, D. Arzelier, J. Daafouz., Robust $H_{2}$ performance analysis and synthesis of linear polytopic discrete-time periodic systems via LMIs, System \& Control Letters 56 (2007) $159-166$.

[17] G. Stepan, T. Insperger, Stability of time-periodic and delayed systems - a route to act and-wait control, Annual Reviews in Control 30 (2006) 159-168.

[18] D. Arzelier, D. Peaucelle, C. Farges, Robust analysis and synthesis of linear polytopic discretetime periodic systems via LMIs, in: Proceedings of the 44th IEEE Conference on Decision and Control, Spain, 2005.

[19] J. Daafouz, P. Riedinger, C. Iung, Stability analysis and control synthesis for switched systems: a switched Lyapunov function approach, IEEE Transactions on Automatic Control 47 (11) (2002) 1883-1887. 
[20] A. Kruszewski, T. M. Guerra, Stabilization of a class of nonlinear model with periodic parameters in the Takagi-Sugeno form, in: Proceedings of the IFAC Workshop Periodic Control Systems, Saint Petersburg, Russia, 2007, pp. 1-6.

[21] H. Kerkeni, T. M. Guerra, J. Lauber, Individual exaust gas mass flow estimation using a periodic observer design, in: Preprints of the IFAC World Congress, Milano, Italy, 2011.

[22] R. E. Skelton, T. Iwasaki, K. Grigoriadis, A Unified Approach to Linear Control Design, Taylor \& Francis, 1998.

[23] H. Tuan, P. Apkarian, T. Narikiyo, Y. Yamamoto, Parameterized linear matrix inequality techniques in fuzzy control system design, IEEE Transactions on Fuzzy Systems 9 (2) (2001) $324-332$.

[24] H. Wang, K. Tanaka, M. Griffin, An approach to fuzzy control of nonlinear systems: stability and design issues, IEEE Transactions on Fuzzy Systems 4 (1) (1996) 14-23. 